\title{
Modeling the Optical System of NASA's EXCLAIM Mission
}

\author{
Gage L. Siebert ${ }^{1}$, Trevor M. Oxholm ${ }^{1}$, Thomas M. Essinger-Hileman² Peter T. Timbie ${ }^{1}$, \\ Eric R. Switzer ${ }^{2}$, and Jason Glenn ${ }^{2}$ \\ ${ }^{1}$ Physics Department, University of Wisconsin-Madison, Madison, WI, USA \\ ${ }^{2}$ NASA Goddard Spaceflight Center, Greenbelt, MD, USA
}

\begin{abstract}
NASA's EXperiment for Cryogenic Large-Aperture Intensity Mapping (EXCLAIM), is a balloon-borne instrument that is designed to perform intensity mapping of $\mathrm{CO}$ and $\mathrm{CII}$ at redshifts as high as $z=3.5$. It will map galaxy and star formation history over cosmological time. EXCLAIM will also demonstrate novel integrated silicon spectrometers using microwave kinetic inductance detectors (MKIDs) that could be an enabling technology for the Origins Space Telescope or other future mid- to far-infrared space telescopes. Here we present progress toward a full electromagnetic simulation of EXCLAIM's optical system at its operational frequencies. The goal of these simulations is to verify the design of the optical system meets mission requirements. When combined with measurements of the instrument, the simulation will also verify that our understanding of the optical system is complete. This work is being done in preparation for the first flight in mid 2022.
\end{abstract}

\section{Introduction}

The rate of star formation over the course of the Universe is poorly understood [Madau \& Dickinson(2014)]. In regions of star formation, gas collapses and cools. This cold molecular gas contains CO, and the ionized regions where star formation has ionized the gas contains CII. Together, they can be used to trace the gas needed to form stars and the star formation itself. CII's fine structure transition from ${ }^{3} P_{3 / 2}$ to ${ }^{3} P_{1 / 2}$ emits at $158 \mu \mathrm{m}(1.90 \mathrm{THz})$ and $\mathrm{CO}$ has rotational transitions that emit from 460 to $805 \mathrm{GHz}$. EXCLAIM aims to map CO and CII at $420-540 \mathrm{GHz}$; probing redshifts up to $z=3.5$, it will map galaxy and star formation history over cosmological time [Switzer et al.(2021)]. EXCLAIM will use cryogenics to reduce the noise levels of the optical system and a balloon to rise above most of the atmosphere. EXCLAIM will perform intensity mapping, which refers to a method of astronomical observation where the angular resolution is too low to resolve single sources. Instead, a large area is observed and statistical properties are derived from the observed intensity pattern. For EXCLAIM to perform as expected, we wish to achieve an illumination of $-40 \mathrm{~dB}$ or lower on the edge of the primary mirror. We are thus simulating the current design of the optical system to achieve this goal. The results of these simulations can inform the design of the telescope and cement our understanding of the chosen optical system.

The current design of the EXCLAIM optical system, depicted in Figure 1, consists of numerous baffles, filters, and a flat reflector. To simulate this optical system, it is advantageous to consider the equivalent time-reversed case; that is, we consider light emitted from our detectors and then track how it moves through the optical system out to the sky. The optical beam starts at the focal plane containing six MKIDs. It then passes through a lens before reflecting off the secondary mirror. The region between the lens and the secondary mirror contains many baffles and filters and will be hereafter referred to as the baffle tube. Soon after reflecting off the secondary mirror, the optical beam reaches the intermediate focus, at which point there is another baffle. Then the optical beam moves out to the flat reflector known as the folding-flat mirror. After reflecting off the folding-flat mirror, the optical beam travels to the primary mirror where it is reflected out to the sky.

\section{Modeling}

2.1. POPPY POPPY is an open-source optical propagation Python package originally developed for the James Webb Space Telescope [Perrin et al.(2012)]. We are using POPPY to make calculations for an on-axis model of EXCLAIM's optical system. The on-axis model uses lenses with the focal lengths and radii of the mirrors. POPPY cannot straightforwardly account for departures from radial symmetry from the off-axis mirrors; it approximates the 


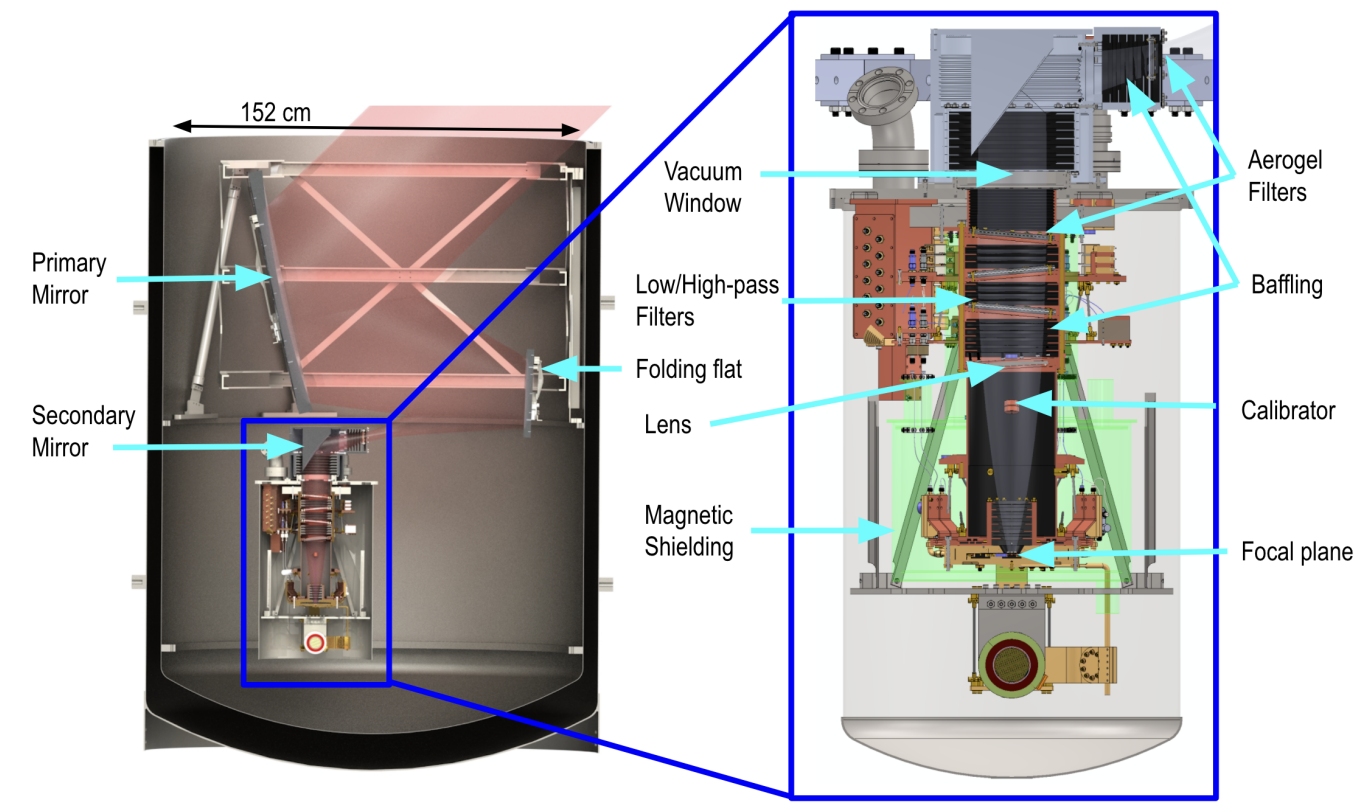

Figure 1: Overview of EXCLAIM's optical system. POPPY simulates the region between the lens and the secondary mirror while CST's simulations begin before the secondary mirror and end at the primary mirror.

optical system with a radially symmetric analogue. We begin our POPPY simulations with a Gaussian beam at the exit of the lens. POPPY handles baffles by simply truncating the beam at the appropriate locations and radii. The output of the POPPY simulations are 2D intensity and phase profiles at any chosen point in the optical system.

2.2. CST Computer Simulation Technology, CST, is a proprietary electromagnetic simulation software. We use CST's Integral Equation Solver, which utilizes the method of moments and the multi-level fast multipole method. The excitation signal is produced using a Near-Field Source, NFS. This NFS is a box with complex electromagnetic fields defined on its surface. The geometry of the box and the field values can be the output of another simulation or user-specified. The output from the simulations are captured on field monitors. These are user-defined planes on which the complex electromagnetic fields are calculated, and from which we can calculate flux. We take the primary illumination by placing field monitors on the plane tangent to the face of the primary mirror.

CST's Integral Equation Solver splits the surfaces of objects into many mesh cells whose sizes are chosen relative to the simulation wavelength. This causes the required memory to go up roughly as simulation frequency squared. This led us to use simulations at relatively low frequencies for testing convergence and consistency. We model the mirrors as perfect electrical conductors but we cannot make this approximation for the baffles. The baffles need to be modeled as a dielectric, which CST then needs to mesh as a volume instead of a surface. This has proved computationally impractical, so we can not straight-forwardly include the baffles in our CST model. For a modest increase in computational requirements, we can approximate a baffle within CST by breaking the simulation in two at the plane of the baffle. We can take the fields on the plane of the baffle from the first simulation, manually truncate them as the baffle would, and insert them into an NFS as an excitation source for the second simulation.

2.3. Combined Model To optimize our simulation of EXCLAIM's optical system, we utilized both simulation software packages: POPPY and CST. CST is the most accurate but it cannot handle some of our absorptive optical elements in a computationally feasible way. Notice the absence of baffles in Figure 2. Thus we have used POPPY to explore baffling configurations, which would be too computationally intensive for CST. The POPPY simulations include the baffle tube, which we expect to have a significant impact on the illumination of the primary [EssingerHileman et al.(2020)].

To minimize the area that CST must mesh, we do not include the folding-flat in our simulations. Instead, we mirrored 


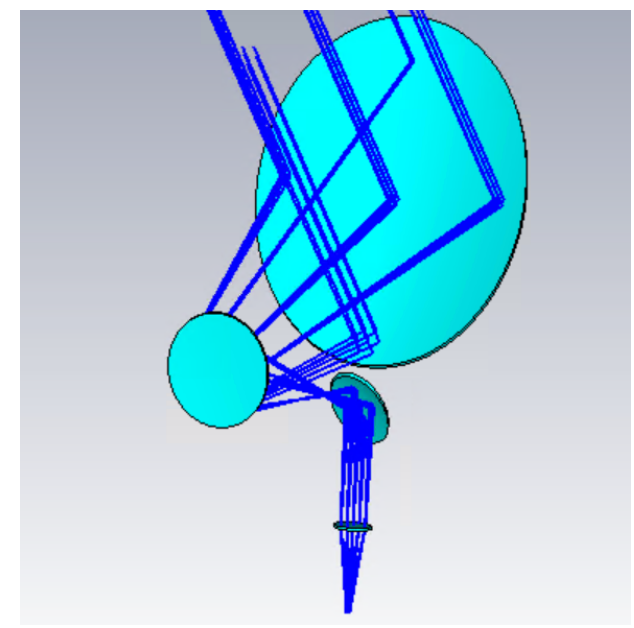

Figure 2: The CAD model of EXCLAIM's mirrors as rendered in CST. The dark blue lines trace the optical beam. The light blue objects are, from top to bottom: the primary mirror, the folding-flat, the secondary mirror, and the lens. The focal plane is at the bottom where the dark blue rays converge.

the primary mirror over the reflective face of the folding-flat. The analogous effect of this simplification in our POPPY simulations is to not truncate at the edge of the folding flat mirror, which we have found to be negligible. At the input to the tertiary mirror, we create an NFS with field values taken from the output of POPPY simulations. We assume that the ratio of field strengths is that of light. We have done tests to show that the polarization of the NFS has little effect on the final result of the CST simulation and so we assume a single linear polarization for simplicity.

\section{Status}

Understanding the way CST simulation settings relate to simulation accuracy has been a high priority. CST has many settings that influence accuracy making this a high dimensional space that is computationally costly to explore. Many test simulations have been required to build an understanding of CST's accuracy settings in the context of our model. We chose to run much of these tests at lower frequencies such as $50 \mathrm{GHz}$. The advantage of testing the simulations at lower frequencies is two-fold. It is easier to attain the computational resources to run these simulations and they also run much faster.
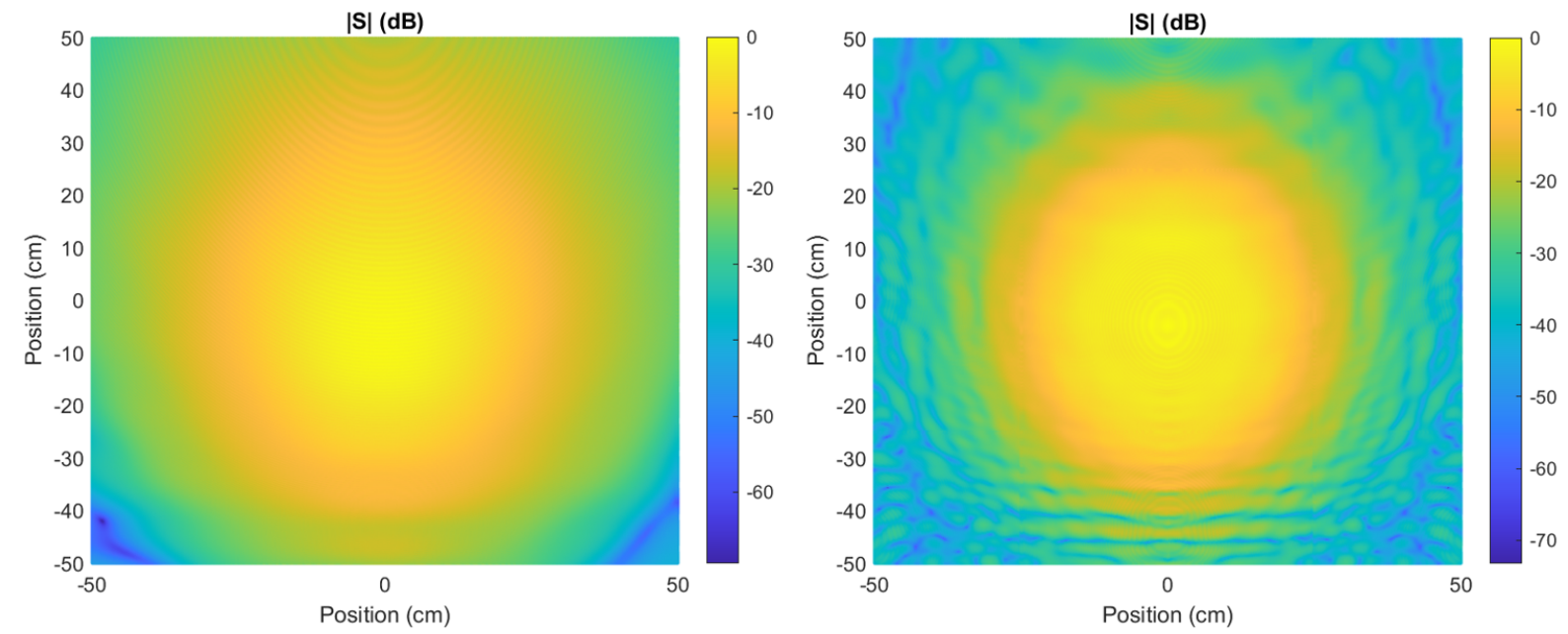

Figure 3: The primary illumination in $\mathrm{dB}$ as calculated with CST at (Left) $50 \mathrm{GHz}$ and (Right) $200 \mathrm{GHz}$. For reference, the primary mirror has a nominal radius of $40 \mathrm{~cm}$.

To date, we have successful CST simulations at frequencies from $50 \mathrm{GHz}$ to $200 \mathrm{GHz}$. We see a modest departure 
from radial symmetry in these primary illuminations. The broadest beams belong to the lowest frequency simulations because diffraction worsens as wavelength increases.

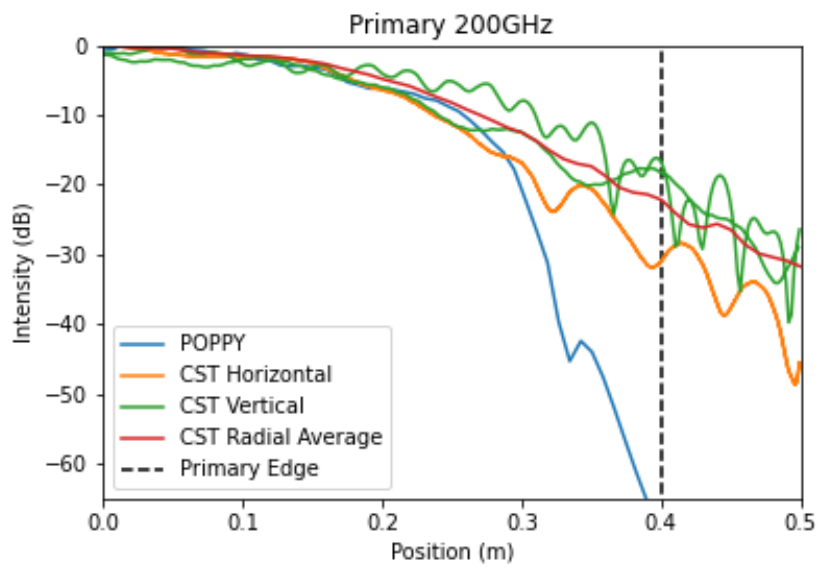

Figure 4: Edge illumination on the primary mirror in analogous POPPY and CST simulations. The vertical cut through the CST beam is taken in the plane of symmetry of the optical system. The horizontal cut is orthogonal to the vertical cut.

Figure 4 shows that CST and POPPY have excellent agreement on primary illumination in the main beam, out to a radius of about $25 \mathrm{~cm}$. It also shows that CST predicts a primary mirror edge illumination that is more than $20 \mathrm{~dB}$ brighter than what POPPY predicts. The errors for the CST values that can be attributed to convergence have been found to be less than $1 \mathrm{~dB}$.

3.1. Challenges The primary illuminations produced by CST are similar to what one would expect if the baffle tube had not truncated the beam. However the baffle tube is included in the POPPY simulation whose output produces the excitation source for CST. The source of this discrepancy is not yet understood. We claim that the difference cannot be explained by convergence issues alone; we can replicate these results with less accurate simulations.

Completing an accurate CST simulation of EXCLAIM's optical system at frequencies of $420 \mathrm{GHz}$ or higher is challenging, in part, because of the resources required. For results that are accurate enough to determine if the design meets performance goals, we have found that six mesh cells per wavelength are needed. Without the many simplifications we have introduced into our model, simulations above $420 \mathrm{GHz}$ would require terabytes of memory. Additionally, since we are utilizing the University of Wisconsin-Madison's Center for High Throughput Computing, each simulation must finish in under two weeks. Currently, this time constraint is more restrictive than the memory usage.

A field monitor-related issue has also arisen in our high memory simulations. In these simulations, CST seems to index the field monitor data incorrectly, which makes them useless. In an attempt to circumvent this, we have broken our field monitors into many smaller ones that together cover the desired area. This workaround allowed us to create a successful $200 \mathrm{GHz}$ simulation, but we have not yet been successful at frequencies of $300 \mathrm{GHz}$ or higher.

\section{Summary and Discussion}

In an effort to better understand diffraction in the optical system of EXCLAIM, we have developed a computationally feasible model of the optical system and built a robust understanding of CST's convergence criteria in the context of this model. This model uses both POPPY and CST in the regions of the optical system where each is preferable. It also makes novel usage of CST's NFSs as a manually specified excitation source and promises to capture the off-axis effects that POPPY alone cannot. For ease of computation, we began simulations at $50 \mathrm{GHz}$ before moving upward toward $420 \mathrm{GHz}$. So far, we have successfully reached as high as $200 \mathrm{GHz}$. Though there remain questions about how CST handles the side lobes of the beam, its main lobe results have excellent agreement with expectation. Other computational challenges have delayed advancement above $200 \mathrm{GHz}$. Construction of the mirrors is likely to begin in the coming months, though we still hope to create a successful $420 \mathrm{GHz}$ simulation before this time. Regardless, such a simulation will prove critical to our understanding of EXCLAIM's optical system. 


\section{Acknowledgments}

We utilized computational resources and expertise from the University of Wisconsin-Madison's Center for High Throughput Computing. This work was funded by the Wisconsin Space Grant Consortium Award \#UGR21_6.0.

\section{References}

T. Essinger-Hileman, T. Oxholm, G. Siebert, et al., "Optical design of the EXperiment for Cryogenic Large-Aperture Intensity Mapping (EXCLAIM)," Society of Photo-Optical Instrumentation Engineers (SPIE) Conference Series, $11453,114530 \mathrm{H}(2020)$.

P. Madau \& M. Dickinson, "Cosmic Star-Formation History", Annual Review of Astronomy and Astrophysics, 52, 415-486 (2014)

M. Perrin, R. Soummer, E. Elliott, et al., "Simulating point spread functions for the James Webb Space Telescope with WebbPSF", Space Telescopes and Instrumentation 2012: Optical, Infrared, and Millimeter Wave. Proceedings of the Society of Photo-Optical Instrumentation Engineers (SPIE), 8442, 84423D (2012)

E. Switzer, E. Barrentine, G. Cataldo, et al., "Experiment for cryogenic large-aperture intensity mapping: instrument design", Journal of Astronomical Telescopes, Instruments and Systems 7, 044004 (2021) 